\title{
Is a monkey malaria from Bomeo an emerging human disease?
}

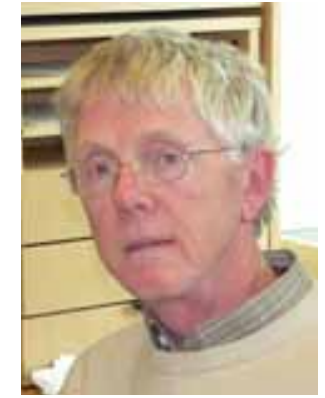

Thomas F M cC utchan

$N$ ational Institute of Allergy $\&$ Infectious D iseases, $N$ ational Institutes of $\mathrm{H}$ ealth, Bethesda, M A 20892-0425, USA

Tel.: +1 301496 6149;

Fax: +1 301402 2201; tmccutchan@niaid.nih.gov future, part of fso
Imedicine

\author{
'The medical community is \\ now asking whether $P$. knowlesi is \\ a "fifth human malaria " with the \\ potential to spread to otherparts \\ of Southeast Asia and beyond.'
}

In 2004, a team of researchers from M alaysia surprised the medical community by reporting that a significant number of people on the island of Borneo were becoming infected with a malaria parasite of monkeys, Plasmodium knowlesi [1]. The world was still unaware of the extent of the problem until 2008 when the same research group gave us a clear view of the gravity of the situation [2]. They found that P. knowlesi infections accounted for $71 \%$ of the cases of malaria in one regional hospital. Of even more concern, the team has shown that this form of malaria is potentially life threatening. The medical community is now asking whether P. knowles is a 'fifth human malaria' with the potential to spread to other parts of Southeast Asia and beyond.

$M$ any species of malaria parasite that naturally infect monkeys will also develop in humans. As early as the 1930s, human infections by monkey malarias were studied under controlled conditions in a number of laboratories, including extensive studies carried out in the USA and Romania. These studies showed that although the infections were not initially life threatening, they became increasingly virulent when cycled through humans in the absence of an intermediate passage through mosquitoes [William C, Pers. Comm] [3]. The virulence associated with the $M$ alaysian infections raises the possibility that a new and more virulent strain of P. knowles is involved as well as the possibility that, in the cases of more virulent infection, some means of parasite transmission other than that initiated by the bite of an infected mosquito is playing a role.

First reported in the Lancet in 2004, Singh and collaborators described their investigation of 208 cases of malaria from the Kapit division of $M$ alaysian Borneo [1]. Their interest had arisen because of the severity of the disease in patients that had been diagnosed as being infected with Plasmodium malariae, which causes a relatively benign form of human malaria. They found that over half of these cases were the result of infection with a monkey malaria, P. knowlesi. In 2008 , the group published the results of a more extensive study on the distribution of human infection by P. knowles in Sarawak and Sabah in Borneo and Pahang peninsular M alaysia. They demonstrated that these infections are occurring in significant numbers in many of the district hospitals. Furthermore, their investigation of four deaths in the region indicated high levels of infection with P. knowles and hepatorenal dysfunction, which is often associated with high parasitemias. This suggested that infection with P. knowlesi is potentially life threatening. Singh, Cox-Singh and Conway now proceed with their ground-breaking research on several fronts, including epidemiological studies to unravel the complicated story of how this emerging disease is being transmitted and further investigation of fatalities in the region to determine the extent to which the threat presents to the people of their region.

'Although mic rosc opy is still the "gold standard" for malaria diagnosis, the morpholog ic al simila rity between $P$. malariae and $P$. knowlesi makes it diffic ult to distinguish between the two.'

There is a sense of urgency associated with these investigations. The rapid replication cycle of P. knowles suggests that the malaria it causes progresses rapidly and that the time involved in identifying the source of infection is critical. Although microscopy is still the 'gold standard' for malaria diagnosis, the morphological similarity between $P$. malariae and P. knowles makes it difficult to distinguish between the two. "Even the best of us see what we anticipate seeing", says Singh. "We are told that there are three human malarias in Malaysia". If a microscopist determines that the infection is not the result of the two most common human parasites, Plasmodium falciparum or Plasmodium vivax, they 
assume that the infection is from the third species, P. malariae. The problem here is that a diagnosis of Plasmodium malaria does not call for immediate and aggressive treatment, while infection with P. knowlesi does.

Scientists now wonder how such virulent malaria infections could have been missed in the past if they occurred to the extent being observed today. More than 50 years ago, the $\mathrm{NIH}$ established a laboratory in peninsular $M$ alaysia for the very purpose of finding monkey malarias in humans. Investigation of the parasites infecting monkeys in the area revealed not only P. knowlesi but also at least five other species of malaria parasites. The researchers were well aware of the difficulty in distinguishing human and monkey parasites by microscopy and proceeded the only way that was available to them. In an effort to determine whether these parasites were being passed to man, blood was taken from approximately 1000 people and put into 200 rhesus monkeys [William C, Pers. Comm]. At the time, this would have been the only way to sort human parasites, which would not grow in the rhesus monkeys, from monkey parasites that would. The procedure would be considered extremely sensitive even by present standards. The conclusion at the time was that monkey malaria infections in humans were not occurring to any significant extent on the peninsula. $\mathrm{N}$ ow, scientists wonder whether they were not occurring or whether the researchers were not looking in the right place. The researchers investigating infections on the island are now armed with modern molecular techniques, such as PCR. This has allowed them to rapidly investigate large numbers of samples and identify the infectious agent definitively, without laborious and expensive experiments with monkeys. Still, the technology is no more sensitive and the results make one ask again whether these infections represent something new.

'Scientists now wonder how such virulent malaria infections could have been missed in the past if they occurred to the extent being observed today.'

There is little doubt that P. knowlesi can be transmitted to humans by the bite of an infected mosquito $[4,5]$. I ronically, one of the investigators sent to peninsular Malaysia was infected with P. knowlesi, presumably the result of mosquito transmission on the peninsula, shortly after he returned to the US. O ne would expect, however, that the infections would be infrequent and that the resulting disease would be relatively mild. The mosquito species, Anopheles latens, which effectively transmits P. knowles in Borneo, inhabits the upper canopy of the forests [6]. Their contact with humans would be thought to be minimal and there is little reason to think that has changed much over the past 50 years.

'It is a lways diffic ult to decipher c a use-and-effect relationships when a nalyzing changes in ma la ria endemnic ity. Parasite, vector and environment a re so intimately tied that changes in any one can affect the disease.'

The geographic distribution of infections is still unknown. "We see only the cases admitted to the hospitals in Borneo", says Singh. "We do not know whether it is reflective of what is happening on the peninsula or elsewhere". Individual cases of P. knowlesi infection in humans have been found in other areas of Southeast Asia but there have been no other clustering of cases. To date, this occurs only in regions where the biological ranges of humans, monkeys and select species of mosquitoes (Anopheles leucosphyrus group) overlap. M embers of the A. leucosphyrus group live in peninsular $M$ alaysia as well as other places in Southeast Asia and so one must ask, why here and why now?

It is always difficult to decipher cause-andeffect relationships when analyzing changes in malaria endemnicity. Parasite, vector and environment are so intimately tied that changes in any one can affect the disease. There has been one dramatic change in the region over the past several years, however, that may have had an impact on the present situation. The $M$ alaysian government has had great success with their malaria control programs, reducing the number of infections by $90 \%$ in some areas. Cox-Singh suggests that the successful control of human malarias may have allowed P. knowlesi to float to the surface. This is a very insightful idea. The number of coinfections with multiple species in a given area is often significantly lower than would be expected if the parasites were acting independently. It has been established for nearly 80 years that one species of malaria parasite will suppress the growth of another species and that coinfection of humans with multiple species can affect the course of the disease. Although the mechanism of suppression is unknown, it has 
been suggested that this could occur through 'heterologous immunity', that is, immunity raised by one species that acts on other species. In fact, this idea could provide a simple answer for the apparent proliferation of P. knowlesi infections in Borneo.

Another concern raised by this work relates to malaria control. We have no way of eradicating a malaria that has an animal reservoir because no matter how effectively we protect humans the transmission cycle will continue in monkeys. Furthermore, the mosquitoes that carry the disease have very different habits than those that transmit human malaria parasites. For example, they feed outside of houses, nullifying the effects of bed nets and the indoor use of insecticides. If Cox-Singh's suggestion that malaria control efforts have allowed P. knowlesi an opportunity to surface, we may find that they have uncovered a new problem for malaria eradication.

The possibility that direct blood transfers between people play a role in the present situation should not be discounted, nor should the possibility that it may play a rolein disease transmission in the future. The direct transmission of blood-borne disease between humans is well established and frequently happens in hospitals but could happen in any number of ways, including in the preparation of hunted game, such as monkeys. It should be remembered that there was an epidemic of malaria in N ew York City (USA) shortly after World War II. Work by the $C D C$ revealed that malaria cases in the city had nothing to do with mosquito transmission but were the result of blood transfer relating to heroin use [7]. Direct transfers can provide inoculums that are hundreds of thousands times larger than the bite of an infected mosquito and would be predicted, as in other direct transfer experiments, to give a more virulent infection. This type of transmission is also more frequently associated with jaundice compared with mosquito-borne transmission [8] and, coincidentally, was symptomatic of all four deaths investigated in Borneo. With regard to the future, the concern related to expansion of this disease through the blood supply is a very real one. For M alaysia and much of Southeast Asia, protecting the blood supply from this new threat becomes imperative. At present no country tests its blood supply for the presence of P. knowlesi.

There are still a number of questions that remain difficult to resolve. $O$ ne of the most intriguing is the fact that the monkeys that carry P. knowles are frequently infected with other species of malaria parasites. A number of these are known to develop in humans and be associated with malaria pathogenesis. For example, both Plasmodium innui and Plasmodium cynomolgi infect humans. These monkey parasites would be predicted to have a better opportunity to infect humans than P. knowlesi because they are transmitted by mosquito vectors that frequently bite man and transmit human malaria. If $P$. knowles infections are being found, why are we not also seeing infections by these parasites?

'For Malaysia and much of Southeast Asia, protecting the blood supply from this new threat becomes imperative. At present no country tests its blood supply for the presence of $P$. knowlesi.'

N ow we wait, as we do with the avian flu, to see if $P$. knowlesi represents a pathogen which has taken the 'jump' to a new host. The distinction between a disease that is occasionally transmitted to humans who venture into an area of natural transmission (zoonosis) and an actual human disease, is one with serious biological implications. If P. knowles is in fact a 'fifth human malaria', the parasite would not only have to be transmissible from one human to another but also be transmitted to the extent that a stable transmission cycle could be maintained in the absence of macaques. This is the case with the four known human malarias. The distinction has enormous ramifications for our understanding of how the disease could expand. Singh and Cox-Singh caution that, as yet, they have no proof of human-to-human transmission. At this point, the study of P. knowlesi is extremely significant regardless of whether it has entered humans permanently or represents a zoonosis. In either case, we face a heath problem of potentially widespread significance and one that will present new problems for malaria control.

Financial \& competing interests disclosure

The author has no relevant affiliations or financial involve ment with any organization or entity with a financial interest in or financial conflict with the subject matter or materials discussed in the manuscript. This includes employment, consultancies, honoraria, stock ownership or options, expert tesimony, grants or patents received or pending, or royalties

No writing assistance was utilized in the production of this manuscript. 


\section{Bibliography}

1. Singh B, Kim Sung L, M atusop A et al.: A large focus of naturally acquired Plasmodium knowles infections in human beings. Lancet 363(9414), 1017-1024 (2004).

2. Cox-Singh J, D avis TM, Lee KS et al.: Plasmodium knowlesi malaria in humans is widely distributed and potentially life threatening. Clin. Infect. D is. 46(2), 165-171 (2008).

3. Ciuca M, Lupasco G, N egulici E, Constantinesco P: Research on the experimental transmission of Plasmodium malariae to man. Arch. Roum. Pathol. Exp. M icrobiol. 23, 763-776 (1964).
4. Chin W, Contacos PG, Collins WE, Jeter $\mathrm{MH}$, Alpert E: Experimental mosquito-transmissions of Plasmodium knowlesi to man and monkey. Am. J. Trop. M ed. H yg. 17(3), 355-358 (1968).

5. Eyles DE, Coatney GR, G etz M E: Vivax-type malaria parasites of macaques transmissible to man. Science 131, 1812-1813 (1960).

6. Vythilingam I, Tan $\mathrm{CH}$, Asmad M , Chan ST, Lee KS, Singh B: N atural transmission of Plasmodium knowlesi to humans by Anopheles latens in Sarawak, M alaysia. Trans. R. Soc. Trop. M ed. Hyg. 100(11), 1087-1088 (2006).
7. M ost H : M anhattan: 'a tropical Isle'? Am. J. Trop. M ed. H yg. 17(3), 333-354 (1968).

8. Chalmers $\mathrm{T}$ : $T$ he occurrence of jaundice in therapeutic and natural malaria. J. Clin. Invest. 26(6), 1055-1059 (1947).

Affiliation

- ThomasF M cCutchan $N$ ational Institute of Allergy \& Infectious $D$ iseases, $N$ ational Institutes of $H$ ealth, Bethesda, M A 20892-0425, U SA Tel.: +1 301496 6149; Fax: +1 301402 2201; tmccutchan@niaid.nih.gov 